RESPIRATORY INFECTION

\title{
Enhanced virulence, airway inflammation and impaired lung function induced by respiratory syncytial virus deficient in secreted $G$ protein
}

\section{J Schwarze, U Schaver}

Thorax 2004;59:517-521. doi: 10.1136/thx.2003.017343

See end of article for authors' affiliations

\section{Correspondence to:} Dr med J Schwarze Department of Respiratory Medicine, National Heart and Lung Division, Imperial College, London W2 IPG, UK :

i.schwarze@ic.ac.uk

Received 14 October 2003 Accepted

18 February 2004

\begin{abstract}
Background: Respiratory syncytial virus (RSV) infection can cause bronchial hyperresponsiveness and asthma exacerbations. In mice it results in airway inflammation and airway hyperresponsiveness. Since viral factors influencing these responses are not well defined, a study was undertaken to investigate the role of secreted G protein of human RSV in determining virulence, inflammatory responses, and changes in lung function.

Methods: BALB/c mice were infected with a spontaneous mutant of RSV deficient in secreted G protein (RSV- $\Delta s G$ ) or with wild type RSV (RSV-WT). Viral titres, numbers of pulmonary inflammatory cells, and concentrations of interferon (IFN)- $\gamma$, interleukin (IL)-4, IL-5 and IL-10 in bronchoalveolar lavage (BAL) fluid were determined. Airway function was assessed at baseline and following methacholine provocation using barometric whole body plethysmography.

Result: Following infection with RSV- $\Delta \mathrm{sG}$, viral titres were increased 50-fold compared with RSV-WT. Influx of eosinophils and macrophages to the lung and concentrations of IFN- $\gamma$ and IL-10 in BAL fluid were also significantly higher following infection with RSV- $\Delta s G$. Airway function, both at baseline and after methacholine provocation, was significantly decreased following infection with RSV- $\Delta \mathrm{sG}$ compared with RSV-WT.

Conclusion: Secreted G protein is likely to be a regulatory factor in RSV infection limiting infectivity of the virus, inflammatory responses in the lungs, and reduction in lung function.
\end{abstract}

$\mathrm{R}$ espiratory syncytial virus (RSV) infection can result in severe bronchiolitis in infants, in asthma exacerbations, and in bronchial hyperreactivity in non-asthmatic subjects. ${ }^{12}$ The viral factors involved in the development of airway inflammation and in changes of lung function triggered by RSV are not well defined.

RSV G protein is not only expressed bound to membranes but is also secreted into the extracellular environment. ${ }^{3}$ Vaccination with $G$ protein in rodent models resulted in severe pulmonary inflammation with airway eosinophilia on subsequent RSV challenge and was associated with the induction of antigen specific Th2 cells. ${ }^{4}$ From these adverse effects of vaccination it was concluded that the $G$ protein might play a critical role in the development of reactive airway disease in RSV infection. Secreted G protein induced even stronger Th2 responses and airway eosinophilia than the membrane bound form, ${ }^{5}$ suggesting that this protein may represent a strategy to modulate and evade effective immune responses. These vaccination models, however, need not reflect the role of viral proteins in primary RSV infection. G protein is thought to be an attachment protein binding primarily to heparin like glycosaminoglycans. ${ }^{6}$ Using RSV variants with targeted mutations of this protein, it has been shown that the presence of either membrane bound or secreted $G$ protein is sufficient for normal attachment and infectivity, ${ }^{7}$ and that alternative pathways of attachment independent of glycosaminoglycans are used in the absence of membrane bound G protein. This suggests a non-essential accessory role for $\mathrm{G}$ protein in RSV attachment and infection. To date no models have been reported investigating the role of membrane bound or secreted G protein in the development of pulmonary inflammation and changes in airway function in primary RSV infection.
A spontaneous variant of human RSV (RSV- $\Delta s \mathrm{~s})$ with a mutated second start codon of the $G$ protein gene preventing transcription of secreted $G$ protein has recently been characterised. ${ }^{8}$ This mutant replicates well in A549 cells, a human alveolar epithelial cell line, and induces increased production of proinflammatory chemokines and increased activation of the transcription factor $\mathrm{NF \kappa B}$. To delineate the role of secreted $G$ protein for the virulence of RSV in vivo and to investigate mechanisms linking secreted G protein to the development of airway inflammation and altered lung function, we used RSV- $\Delta \mathrm{sG}$ in a murine model of primary infection. Viral titres in the lung, pulmonary inflammation, lung function, and local cytokine production were monitored and compared with infection with wild type RSV (RSV-WT) which results in eosinophil and neutrophil influx into the lung and in the development of interleukin (IL)-5 dependent airway hyperresponsiveness to inhaled methacholine in this model. ${ }^{9}{ }^{10}$

\section{METHODS}

\section{Virus}

Human RSV type A (Long strain) from ATCC (Rockville, MD, USA) and RSV- $\Delta \mathrm{sG}$ from the Department of Medical Virology, Ruhr-Universität (Bochum, Germany), both free of chlamydia or mycoplasma contamination, were cultured in HEp-2 cells (ATCC) and purified by centrifugation using a $30 \%$ sucrose cushion as described. ${ }^{11}$ The resulting pellet was resuspended in phosphate buffered saline (PBS), aliquoted, and frozen at $-70^{\circ} \mathrm{C}$. Virus was titrated by immunoplaque assay as previously described ${ }^{12}$ and titres were expressed as

Abbreviations: BAL, bronchoalveolar lavage; IFN- $\gamma$, interferon- $\gamma$; IL, interleukin; RSV, respiratory syncytial virus 
tissue culture infective dose at $50 \%\left(\mathrm{TCID}_{50}\right)$ according to Krah. ${ }^{13}$ The stock suspension was adjusted to contain $2 \times 10^{7}$ TCID $_{50}$ RSV/ml. HEp-2 cell supernatant free of RSV was treated in the same way. The resulting mock isolate was used for sham infections.

\section{Animals}

Female BALB/C AnNCrl mice aged 8-12 weeks, free of specific pathogens, from Charles River Laboratories (Sulzfeld, Germany) were kept under specific pathogen free conditions and used under a protocol approved by Regierungspräsidium Arnsberg (Germany).

\section{Experimental protocols}

Mice were infected on day 0 under light anaesthesia by intranasal inoculation of $10^{5}$ TCID $_{50}$ RSV-WT or RSV- $\Delta \mathrm{sG}$ in $50 \mu \mathrm{l}$. Controls were sham infected with mock isolate. To assess lung RSV titres, some lungs were removed 4 days after infection and disrupted in $1 \mathrm{ml}$ PBS using a glass homogeniser. Following centrifugation, supernatant of the homogenate was added to HEp-2 cell cultures. TCID 50 was determined as described above. Airway responsiveness was assessed on day 6 after infection and animals were killed the following day for collection of bronchoalveolar lavage (BAL) fluid and removal of lungs. To deplete IL-5, mice were treated intravenously with $150 \mu \mathrm{g}$ anti-IL-5 antibody from clone TRFK-5 (Becton and Dickinson, Heidelberg, Germany) on days 0 and 3 after infection. Controls were injected with $150 \mu \mathrm{g}$ rat $\operatorname{IgG}_{1}$ (SigmaAldrich) at the same time points.

\section{Lung function}

Lung function was assessed using a whole body plethysmograph obtained from Buxco (Troy, NY) as described elsewhere. $^{14}$ In the plethysmograph, mice were exposed for 3 minutes to nebulised PBS and subsequently to increasing concentrations of aerosolised methacholine (Sigma-Aldrich) in PBS using an AeroSonic ultrasonic nebuliser (DeVilbiss, Somerset, PA, USA). After each aerosolisation, enhanced pause (Penh) values were recorded for 3 minutes, averaged, and expressed as absolute values.

\section{Lung cell isolation}

Lung cells were isolated by collagenase digestion as described previously ${ }^{15}$ and counted with a haemocytometer. Cytospin slides were stained with Haema-Schnellfärbung (Labor \& Technik Lehmann, Berlin, Germany) and differential cell counts of 300 cells were performed in a blinded fashion by light microscopy.

\section{Cytokines in BAL fluid}

Tracheas were dissected, lungs lavaged with 1 ml Hank's balanced salt solution (HBSS, Biochrom), and BAL fluid supernatants frozen at $-20^{\circ} \mathrm{C}$. Concentrations of IFN- $\gamma$, IL-4, IL-5, and IL-10 were assessed by Opteia ELISA kits (Becton and Dickinson).

\section{Statistical analysis}

Data were compared using GraphPad Prism 2.01 (GraphPad Software, San Diego, USA ). The Mann-Whitney test was used for comparisons of two groups and the Kruskal-Wallis test with Dunn post hoc test were used for comparisons of more than two groups. The area under the curve of repeated measurements was compared in airway responsiveness assays. $p$ values of $<0.05$ were considered statistically significant. Values are expressed as mean (SE) for all measurements.

\section{RESULTS}

\section{RSV replication in the lung}

To assess the role of secreted G protein for in vivo replication, mice were infected intranasally with RSV- $\Delta s$ G or RSV-WT. In all infected animals tested, infection could be demonstrated but not in mice sham infected with mock isolate. Infection with RSV- $\Delta$ sG resulted in pulmonary virus titres 50 -fold higher than infection with RSV-WT on day 4 after infection (mean (SE) TCID 5050133 (6162) v 970 (471), $\mathrm{n}=6$, $\mathrm{p}=0.02)$.

\section{Airway inflammation}

To investigate changes in pulmonary inflammatory cells during infection, lung cells were isolated 7 days after infection and differential cell counts performed. In mice infected with RSV-WT the numbers of eosinophils and neutrophils were significantly increased in lung cell isolates compared with sham infected controls (fig $1 \mathrm{~A}$ and $\mathrm{B}$ ). Following RSV- $\Delta \mathrm{sG}$ infection the increases in numbers of eosinophils exceeded those induced by RSV-WT (fig 1A). In addition, total lung cell numbers were increased (RSV- $\Delta \mathrm{sG}$ : $52.0 \quad(4.8) \times 10^{6}$; RSV-WT: $39.8 \quad(3.2) \times 10^{6}$; mock: 33.7 $(2.1) \times 10^{6} ; \mathrm{n}=12$, significant difference across all groups $\mathrm{p}<0.001)$ and the influx of macrophages increased 1.8 -fold compared with RSV-WT (fig 1B).

\section{Lung function}

Airway function without provocation (baseline) and airway response to methacholine were assessed on day 6 after infection (all groups $n=12$ ). Mice infected with RSV-WT had increased airway responsiveness to methacholine compared with sham infected controls (fig 2). Maximal Penh in response to $25 \mathrm{mg} / \mathrm{ml}$ methacholine increased 4.7 -fold over PBS in mice infected with RSV-WT compared with a 3.1-fold increase in sham infected mice. Following infection with RSV- $\Delta$ SG (but not with RSV-WT), baseline Penh increased 2.3-fold compared with sham infected controls in the absence of provocation, indicating a reduction in lung function even without exposure to methacholine. Airway responsiveness to methacholine provocation, expressed as the change from baseline, did not differ significantly between mice infected with RSV- $\Delta$ sG or RSV-WT. In contrast, absolute Penh values following methacholine provocation were significantly higher in mice infected with RSV- $\Delta \mathrm{sG}$ than with RSV-WT.

\section{Cytokine production}

Levels of IFN- $\gamma$, IL-4, IL-5, and IL-10 were assessed in BAL fluid collected on day 7 after infection (all groups $n=12$ ). In mice infected with RSV-WT, levels of IFN- $\gamma$ were significantly increased compared with controls (fig 3). Concentrations of IL-5 and IL-10 did not differ from controls. IL-4 was not detectable (data not shown). Following infection with RSV$\Delta \mathrm{sG}$ the levels of IFN- $\gamma$ increased even further and concentrations of IL-10 were also significantly raised (fig 3). The levels of IL-5 were virtually unchanged and IL-4 remained below the level of detection.

\section{Anti-IL-5 treatment}

We have previously shown that both pulmonary eosinophil influx and airway hyperresponsiveness following RSV-WT infection depend on the presence of IL-5. To investigate whether this also holds true for changes induced by infection with RSV- $\Delta s G$, mice were treated during infection with anti-IL-5 antibody. Administration of anti-IL-5 antibody, but not of rat $\operatorname{IgG}_{1}$ as a control, prevented increases in numbers of eosinophils (mean (SE) $0.15 \quad(0.03) \times 10^{6} \quad v \quad 2.23$ $\left.(0.42) \times 10^{6}, \mathrm{n}=12, \mathrm{p}<0.001\right)$ without affecting the influx of neutrophils $\left(15.52(3.77) \times 10^{6} v 17.30(3.55) \times 10^{6}, \mathrm{n}=12\right)$ or 

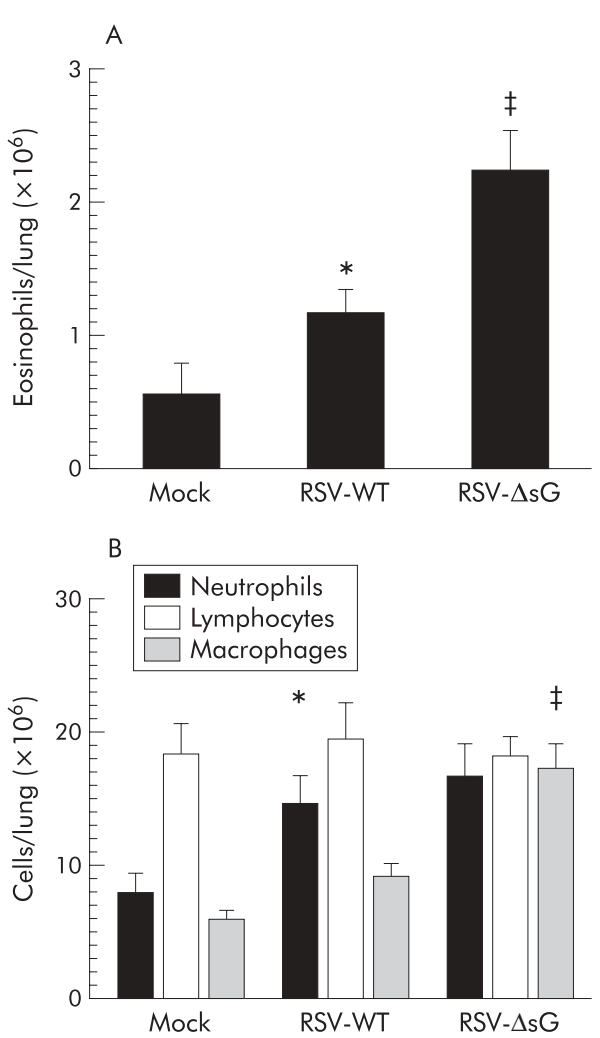

Figure 1 Numbers of pulmonary eosinophils and macrophages following infection with RSV- $\Delta s G$, RSV-WT, or mock isolate ( $n=12$ in each group). Lung cells were isolated 7 days after infection and numbers of (A) eosinophils and (B) neutrophils, lymphocytes and macrophages per lung were determined. Data shown are mean (SE) cell numbers from three independent experiments. Significant difference across all groups $p<0.001$. *RSV-WT $\vee$ mock; $\ddagger R S V-\Delta s G \vee R S V-W T$.

macrophages $\left(24.78(6.82) \times 10^{6} v 26.76(4.57) \times 10^{6}, \mathrm{n}=12\right)$ following RSV- $\Delta \mathrm{sG}$ infection.

Furthermore, depletion of IL-5 resulted in a significant reduction in Penh values both at baseline and after methacholine provocation (fig 4), whereas application of rat $I g G_{1}$ did not have a significant effect. In mice infected with RSV-WT, airway hyperresponsiveness was abolished completely by anti-IL-5 treatment (data not shown).

\section{DISCUSSION}

In order to delineate the role of secreted $G$ protein in primary RSV infection and in associated pulmonary inflammation and airway hyperresponsiveness, we studied a spontaneous mutant of RSV lacking the secreted G protein. In this virus a mutation in the second open reading frame of the $G$ protein gene where transcription of secreted $G$ protein is initiated causes an exchange of the amino acid in position 48 ( $M$ to $\mathrm{T}$ ) resulting in the loss of the second start codon of the $G$ protein gene and thus preventing transcription of its secreted form. ${ }^{8}$ RSV- $\Delta \mathrm{sG}$ was used in an established mouse model of RSV infection and viral titres in the lungs, pulmonary inflammation, lung function, and local cytokine production were monitored. In this model, RSV-WT infection results in airway hyperresponsiveness, pulmonary inflammation with influx of eosinophils and neutrophils, and increased production of Thl cytokines. ${ }^{9}$

Viral titres in the lungs following infection with RSV- $\Delta s G$ were 50-fold higher than after infection with RSV-WT, indicating that RSV- $\Delta \mathrm{sG}$ caused more efficient infection or replication in vivo. In contrast, in A549 cells, a human type 2

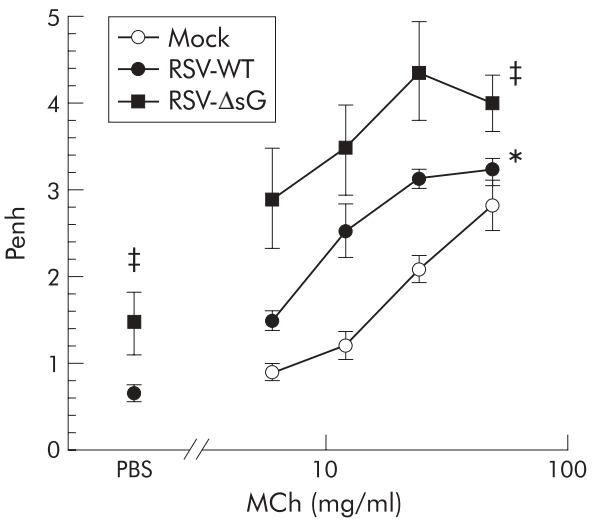

Figure 2 Enhanced pause (Penh) values at baseline and following methacholine (MCh) provocation in mice infected with RSV- $\Delta s G$, RSVWT or mock isolate ( $n=12$ in each group). On day 6 after infection baseline readings and airway responsiveness to increasing concentrations of inhaled MCh $(3-50 \mathrm{mg} / \mathrm{ml})$ were assessed by barometric whole body plethysmography and Penh values were calculated. Mean (SE) Penh values from three independent experiments are expressed as absolute values. Significant difference across all groups $p<0.0001$. * RSV-WT $v$ mock, $\ddagger$ RSV- $\Delta s G \vee R S V-W T$.

alveolar epithelial cell line, replication of RSV- $\Delta \mathrm{sG}$ did not differ from RSV-WT. ${ }^{8}$ A targeted mutation of RSV with a deletion of the secreted $G$ protein was not attenuated either in vitro. ${ }^{7}$ Considering the lack of mutations relevant for replication in RSV- $\Delta \mathrm{sG},{ }^{8}$ these findings imply that secreted $\mathrm{G}$ protein is not required for effective infection and replication of RSV. On the contrary, our findings in vivo suggest an inhibitory effect of secreted $G$ protein on the virulence of RSV. Such an inhibitory effect of secreted G protein may not be evident in culture because of the high multiplicity of infection used which results in instantaneous infection of the majority of cells. In vivo, probably only a minority of epithelial cells are infected initially. Recently, the fractalkine receptor CX3C-receptor 1 has been identified as a receptor for RSV G protein. ${ }^{16}$ Soluble G protein could limit RSV infection by binding to $\mathrm{CX} 3 \mathrm{C}$-receptor $\mathrm{l}$, thus reducing the availability of this receptor for live virus with membrane bound $G$ protein.

Inflammatory responses to RSV- $\Delta \mathrm{sG}$ were assessed in lung cell isolates. Increases in numbers of total inflammatory lung cells and eosinophils exceeded those observed after RSV-WT infection. Furthermore, infection with RSV- $\Delta s$ G but not with RSV-WT resulted in striking increases in the numbers of pulmonary macrophages. These findings suggest that the inflammation resulting from RSV infection in the absence of secreted G protein is more severe than in RSV-WT infection, and it differs in quality. Such differences in the cellular response could be due not only to the severity of infection but also to the absence of chemokine inhibitory actions of secreted G protein. Secreted glycoproteins can bind to chemokines, inhibiting their activity, as has been shown for poxviruses and Ebola virus. ${ }^{17}{ }^{18}$ The lack of such glycoproteins is associated with increased local inflammation ${ }^{17}$ and with increased cytotoxicity. ${ }^{18}$ These secreted glycoproteins thus serve as protective factors for virus infected cells, subverting the host immune response and limiting inflammation. Secreted G protein might serve the same purpose in RSV infection.

Arnold and colleagues found that, in A549 cells, RSV- $\Delta$ sG induces increased activation of the transcription factor NFKB compared with RSV-WT and consequently leads to increased production of chemokines including RANTES. ${ }^{8}$ Transcription of RANTES is enhanced by $\mathrm{NF \kappa B}^{19}$ and increased production could account for enhanced recruitment of eosinophils and 

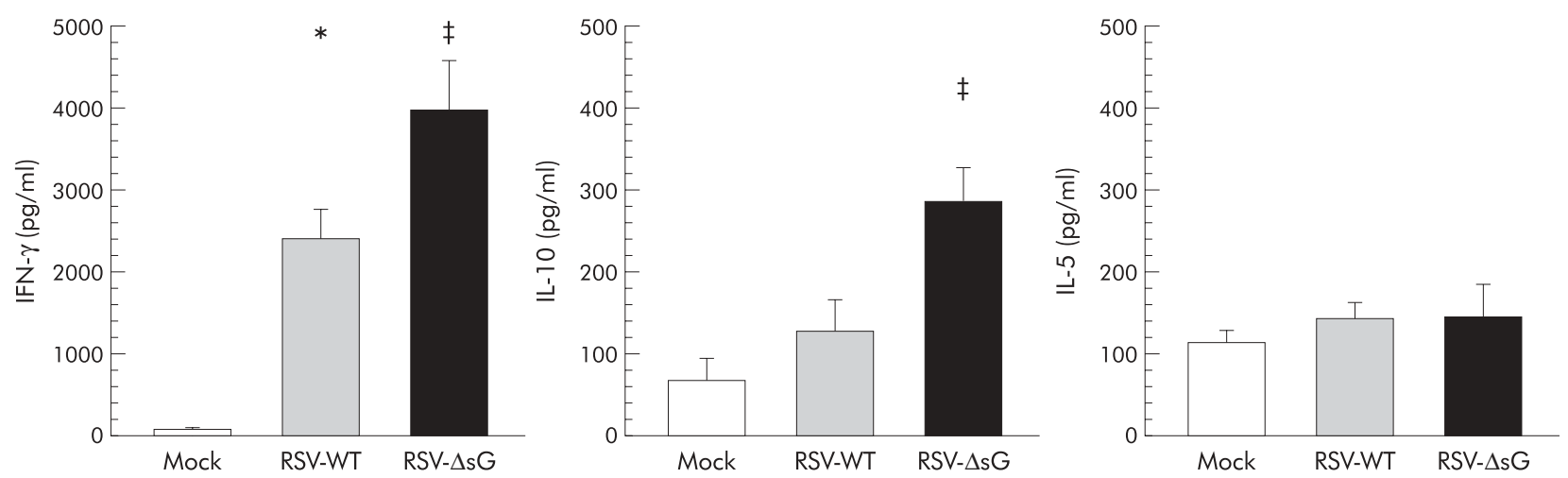

Figure 3 Concentrations of interferon (IFN)- $\gamma$ and interleukin (IL)- 10 and IL-5 in BAL fluid following infection with RSV- $\Delta s G$, RSV-WT, or mock isolate ( $n=12$ in each group). Concentrations of IFN- $\gamma$, IL-10, and IL-5 were determined by ELISA. Mean (SD) cytokine concentrations from three independent experiments are shown. For IFN- $\gamma$ and IL-10, significant difference across all groups $p<0.0001$. *RSV-WT $v$ mock, $\ddagger R S V-\Delta s G \vee R S V-W T$.

macrophages to the lung. They express the RANTES binding CC-chemokine receptors 3 and 5, respectively. ${ }^{20}{ }^{21}$ RANTES, which also attracts Th2 cells, ${ }^{22}$ has recently been shown to be critical for the severity of RSV induced inflammation and airway hyperresponsiveness in mice. ${ }^{23}$ The intercellular adhesion molecule (ICAM)-1 was also upregulated to higher levels following infection by RSV- $\Delta \mathrm{SG}$ than by RSV-WT. ${ }^{8}$ Increased ICAM-1 expression on infected cells also probably contributes to enhanced recruitment of inflammatory cells to the lung. Interestingly, ICAM- 1 binds to the F protein of RSV and may also be involved in the adhesion of RSV to cells and subsequent cell entry. ${ }^{24}$ Thus, increased ICAM-1 expression induced by RSV- $\Delta \mathrm{sG}$ infection could enhance infectivity of this mutant even further.

Cytokine production in BAL fluid was assessed to characterise the pulmonary immune response to RSV- $\Delta \mathrm{sG}$ infection. Infection with RSV-WT resulted in significant increases in IFN- $\gamma$ levels which were increased further following infection with RSV- $\Delta \mathrm{sG}$. IFN- $\gamma$ is the most abundant cytokine following RSV infection..$^{26}$ IL-10, which is also readily detected in macrophage ${ }^{27}$ and $\mathrm{T}$ cells following

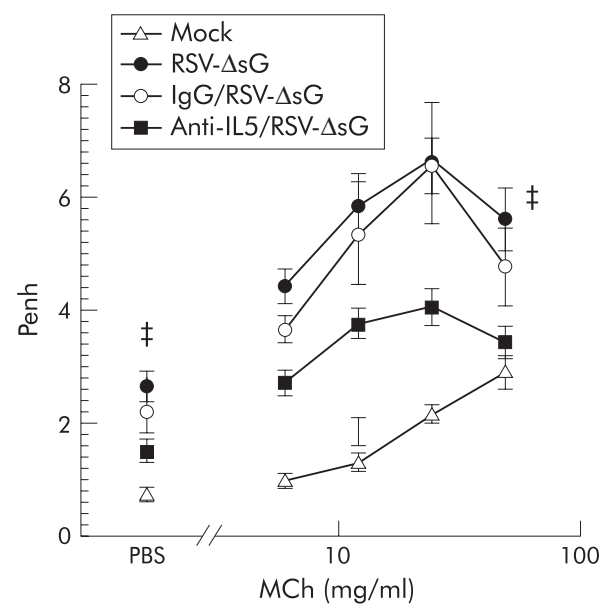

Figure 4 Effect of anti-IL-5 treatment on RSV- $\Delta s G$ induced increases in Penh at baseline and following methacholine (MCh) provocation. Mice were sham infected (mock, $n=9)$ or infected with RSV- $\Delta s G(n=9)$. Some infected mice were treated with anti-IL-5 antibody (anti-IL-5/RSV- $\Delta \mathrm{sG}$, $n=9)$ or with rat $\lg G(\lg G / R S V-\Delta s G, n=9)$ as a control. On day 6 after infection baseline readings and airway responsiveness to increasing concentrations of inhaled MCh $(3-50 \mathrm{mg} / \mathrm{ml}$ ) were assessed and Penh values were calculated. Mean (SE) Penh values from three independent experiments are expressed as absolute values. Significant difference across all groups $p<0.0001 . \ddagger R S V-\Delta s G \vee$ anti-IL-5/RSV- $\Delta s G$.
RSV infection, ${ }^{25}{ }^{26}$ remained low following infection with RSV-WT but was significantly increased following infection with RSV- $\Delta$ sG. No increases in IL-4 or IL-5 were observed. Thus, cytokine responses to RSV- $\Delta \mathrm{sG}$ and RSV-WT differ in intensity but not in quality, suggesting that secreted G protein is inhibitory for immune responses but no major determinant for Th1/Th2 polarisation in primary RSV infection. This is in contrast to findings following vaccination with secreted $G$ protein where it serves as a potent immunogen inducing strong Th2 polarisation on subsequent RSV challenge. ${ }^{5}$

Baseline lung function in the absence of provocation and airway responsiveness to methacholine were monitored using barometric whole body plethysmography. In contrast to infection with RSV-WT where increases in baseline Penh do not occur, ${ }^{910}$ infection with RSV- $\Delta$ sG resulted in a twofold increase in baseline Penh values indicating a decrease in lung function. Furthermore, compared with baseline Penh values, airway responsiveness following methacholine provocation was similar in mice infected with RSV- $\Delta s$ G or RSV-WT. However, absolute Penh values following exposure to methacholine were significantly higher in RSV- $\Delta \mathrm{sG}$ than in RSV-WT infection. Increases in Penh values following methacholine provocation have been shown to correlate closely with increases in lung resistance in mice in both models of allergen induced airway inflammation ${ }^{14}$ and of RSV infection (unpublished data). The increases in Penh values observed here therefore suggest more severe airway obstruction following methacholine exposure in RSV- $\Delta \mathrm{sG}$ infected mice.

We have previously shown that IL-5 has a critical role in RSV induced airway hyperresponsiveness. ${ }^{10}$ To investigate whether RSV- $\Delta$ sG induced increases in Penh values at baseline and following methacholine provocation are also dependent on IL-5, mice were treated with anti-IL-5 antibody during infection. This treatment prevented increases in the numbers of pulmonary eosinophils and significantly reduced increases in Penh values both at baseline and after methacholine provocation. In mice infected with RSV-WT, airway hyperresponsiveness was abolished completely by anti-IL-5 treatment as previously shown. ${ }^{9}{ }^{10}$ These results indicate that decreases in lung function following RSV- $\Delta \mathrm{sG}$ infection are only partially dependent on IL-5 (and possibly on eosinophils) and that other mechanisms are probably also involved. Increased production of IL-10 from increased numbers of pulmonary macrophages could contribute to RSV- $\Delta \mathrm{sG}$ induced changes in lung function. In a model of allergic airway inflammation the presence of IL-10 was a prerequisite for the development of airway hyperresponsiveness ${ }^{28}$ and 
increases in endogenous IL-10 led to increased airway reactivity.

\section{ACKNOWLEDGEMENTS}

The authors thank $\mathrm{H}$ Werchau for providing RSV- $\Delta \mathrm{sG}$, C Roder for assessing RSV titres in the lung, and Uta Wiedersprecher for technical assistance.

\section{Authors' affiliations \\ J Schwarze, U Schaver, Children's Clinic, St Josef-Hospital, 44791 \\ Bochum, Germany}

J Schwarze, Department of Respiratory Medicine, National Heart and Lung Division, Imperial College, London W2 IPG, UK

This work was supported by grant 01GC9802 from Bundesministerium für Bildung und Forschung and grant AL 067454 from The Wellcome Trust, both to JS.

\section{REFERENCES}

1 Busse WW. Respiratory infections: their role in airway responsiveness and the pathogenesis of asthma. J Allergy Clin Immunol 1990;85:671-83.

2 Cypar D, Stark J, Lemanske RF Jr. The impact of respiratory infections on asthma. Pediatr Clin North Am 1992;39:1259-76.

3 Hendricks DA, Baradaran K, Mclntosh K, et al. Appearance of a soluble form of the $G$ protein of respiratory syncytial virus in fluids of infected cells. J Gen Virol 1987:68:1705-14.

4 Alwan WH, Kozlowska WJ, Openshaw PJM. Distinct types of lung disease caused by functional subsets of antiviral T cells. J Exp Med 1994;179:81-89.

5 Johnson TR, Johnson JE, Roberts SR, et al. Priming with secreted glycoprotein $G$ of respiratory syncytial virus (RSV) augments interleukin-5 production and tissue eosinophilia after RSV challenge. J Virol 1998;72:2871-80.

6 Krusat T, Streckert HJ. Heparin-dependent attachment of respiratory syncytial virus (RSV) to host cells. Arch Virol 1997;142:1247-54.

7 Teng MN, Whitehead SS, Collins PL. Contribution of the respiratory syncytial virus $G$ glycoprotein and its secreted and membrane-bound forms to virus replication in vitro and in vivo. Virology $2001 ; 289: 283-96$.

8 Arnold R, König B, Werchau $H$, et al. Respiratory syncytial virus deficient in soluble G-protein induced an increased proinflammatory response in human lung epithelial cells. Immunobiology 2003;208:160.

9 Schwarze J, Hamelmann E, Bradley KL, et al. Respiratory syncytial virus infection results in airway hyperresponsiveness and enhanced airway sensitization to allergen. J Clin Invest 1997;100:226-33.

10 Schwarze J, Cieslewicz G, Hamelmann E, et al. IL-5 and eosinophils are essential for the development of airway hyperresponsiveness following acute respiratory syncytial virus infection. J Immunol 1999;162:2997-3004.
11 Reuman PD, Keely SP, Schiff GM. Comparison of class and subclass antibody response to live and UV-inactivated RSV administered intranasally in mice. J Med Virol 1991;35:198-205.

12 Foster S, Bedford KJ, Gould ME, et al. Respiratory syncytial virus infection and virus-induced inflammation are modified by contaminants of indoor air. Immunology 2003;108:109-15.

13 Krah DL. A simplified multiwell plate assay for the measurement of hepatitis A virus infectivity. Biologicals 1991;19:223-7.

14 Hamelmann E, Schwarze J, Takeda K, et al. Noninvasive measurement of airway responsiveness in allergic mice using barometric plethysmography. Am J Respir Crit Care Med 1997;156:766-75.

15 Oshiba A, Hamelmann E, Takeda K, et al. Passive transfer of immediate hypersensitivity and airway hyperresponsiveness by allergen-specific immunoglobulin (lg) E and $\operatorname{lgG} 1$ in mice. J Clin Invest 1996;97:1398-1408.

16 Tripp RA, Jones LP, Haynes $L M$, et al. CX3C chemokine mimicry by respiratory syncytial virus G glycoprotein. Nat Immunol 2001;2:732-8.

17 Lalani AS, Masters J, Graham K, et al. Role of the myxoma virus soluble CCchemokine inhibitor glycoprotein, $\mathrm{M}-\mathrm{Tl}$, during myxoma virus pathogenesis. Virology 1999:256:233-45.

18 Volchkov VE, Volchkova VA, Muhlberger E, et al. Recovery of infectious Ebola virus from complementary DNA: RNA editing of the GP gene and viral cytotoxicity. Science 2001;291:1965-9.

19 Delgado M, Jonakait GM, Ganea D. Vasoactive intestinal peptide and pituitary adenylate cyclase-activating polypeptide inhibit chemokine production in activated microglia. Glia 2002;39:148-61.

20 Heath H, Qin S, Rao P, et al. Chemokine receptor usage by human eosinophils. The importance of CCR3 demonstrated using an antagonistic monoclonal antibody. J Clin Invest 1997;99:178-84.

21 Simmons G, Clapham PR, Picard L, et al. Potent inhibition of HIV-1 infectivity in macrophages and lymphocytes by a novel CCR5 antagonist. Science 1997;276:276-9

22 Sallusto F, Mackay CR, Lanzavecchia A. Selective expression of the eotaxin receptor CCR3 by human T helper 2 cells. Science 1997:277:2005-7.

23 Tekkanat KK, Maassab H, Miller A, et al. RANTES (CCL5) production during primary respiratory syncytial virus infection exacerbates airway disease. Eur J Immunol 2002;32:3276-84.

24 Behera AK, Matsuse $\mathrm{H}$, Kumar $M$, et al. Blocking intercellular adhesion molecule-1 on human epithelial cells decreases respiratory syncytial virus infection. Biochem Biophys Res Commun 2001;280:188-95.

25 Hussell T, Spender LC, Georgiou A, et al. Th1 and Th2 cytokine induction in pulmonary T cells during infection with respiratory syncytial virus. J Gen Virol 1996;77:2447-55

26 Tripp RA, Moore D, Anderson L. TH(1)- and TH(2)-type cytokine expression by activated $T$ lymphocytes from the lung and spleen during the inflammatory response to respiratory syncytial virus. Cytokine 2000;12:801-7.

27 Panuska JR, Merolla R, Rebert NA, et al. Respiratory syncytial virus induces interleukin-10 by human alveolar macrophages. Suppression of early cytokine production and implications for incomplete immunity. J Clin Invest 1995:96:2445-53.

28 Makela MJ, Kanehiro A, Borish L, et al. IL-10 is necessary for the expression of airway hyperresponsiveness but not pulmonary inflammation after allergic sensitization. Proc Natl Acad Sci USA 2000;97:6007-12. 